\title{
Scada Based Online Circuit Breaker Monitoring System
}

\author{
Raj Lakshmi, Ankit Pandey \\ (Electrical, Suresh Gyan Vihar University, India)
}

\begin{abstract}
This paper presents the online monitoring of circuit breakers used in the transmission and distribution of power system, to connect and disconnect the power flow at the generator, substation, or load location. The circuit Breakers are capable of making, carrying, and breaking currents under normal circuit conditions. The objective of this paper is to investigate feasibility, specify requirement and propose the implementation architecture of an automated circuit breaker monitoring device that enables continuous and reliable system monitoring. It is very essential to implement an online system for efficient circuit breaker monitoring because of many infrequent cases. To improve reliability of the power system through various analysis functions it is necessary to know power system topology configuration. Real time and on-line monitoring seems to be perfect solution for circuit breaker conditions and status monitoring. SCADA is a system used for this purpose. This system monitors status of CB mechanical contacts and transfers it in real time to a central place.
\end{abstract}

Keywords - circuit breaker, monitoring, plc, protocol, scada

\section{INTRODUCTION}

A circuit breaker is automatically operated electrical switch used in power system to the apparatus from damage caused by overloading /short circuit, it break or make current flow through the electrical circuit. Circuit breaker is widely used in power system to control and protect power equipments under the condition of normal overload, short circuit and other faults. Its basic function is to detect the fault and discontinue power flow immediately without interrupting continuity. The action taken by circuit breaker changes the topology of the power system. An automated circuit breaker monitoring system is proposed to monitor circuit breaker's control circuit. By this system it is possible to track the circuit breaker switching sequences and make conclusions about their performances and final outcome. In order to assure circuit breaker can operate; operation department need put a lot of manpower and material resources in regular maintenance. Therefore, the AC and DC circuit breaker on-line monitoring system becomes both the best choice to solve high-voltage circuit breaker monitoring and the necessary premise to implement circuit breaker condition maintenance.

With increasing importance of scada technology in recent years, it is imperative for all to perceive the development and applications of scada system. Be it in traditional industrial monitoring or in the emerging mobile communication, scada system has gradually moved into all aspects of production. SCADA refers to the combination of telemetry and data acquisition. It encompasses the collecting of the information, transferring it back to the central site, carrying out any necessary analysis and control and then displaying that information on a number of operator screens or displays. The required control actions are then conveyed back to the process. The PLC or Programmable Logic Controller is still one of the most widely used control systems in industry. As needs grew to monitor and control more devices in the plant, the PLCs were distributed and the systems became more sharp and smaller in size.

\subsection{Definition and characteristics of scada system}

\section{The introduction of Scada Technology}

According to IEEE definition, scada is the acquisition of data, the processing of those data for use by the operator, and operator control of remote devices are the fundamental building blocks upon which all modern utility control systems are based.

SCADA is an acronym for Supervisory Control and Data Acquisition. SCADA systems are used to monitor and control a plant or equipment in industries. These systems encompass the transfer of data between a SCADA central host computer and a number of Remote Terminal Units (RTUs) .A SCADA system gathers information (such as where the fault in transmission line has occurred), transfers the information back to a central site, then alerts the home station that a fault has occurred, carrying out necessary analysis and control, such as determining if the fault is critical, and displaying the information in a logical and organized fashion. SCADA systems have made use of the infrastructure of the corporate Local Area Network (LAN)/Wide Area Network (WAN), Wireless technologies for monitoring purposes. 


\subsection{The architecture of Scada System}

Scada is a common process automation system which is used to gather data from sensors and instruments located to remote sites and to transmit data at a central site for either controller monitoring process. The scada system consists of:

- One or more field data interface devices, usually RTUs, or PLCs, which interface to field sensing devices and local control switchboxes and valve actuators

- A communications system used to transfer data between field data interface devices and control units and the computers in the SCADA central host. The system can be radio, telephone, cable, satellite, etc., or any combination of these.

- A central host computer server or servers [sometimes called a SCADA Center, master station, or Master Terminal Unit (MTU)]

- A collection of standard and/or custom software [sometimes called Human Machine Interface (HMI) software or Man Machine Interface (MMI) software] systems used to provide the SCADA central host and operator terminal application, support the communications system, and monitor and control remotely located field data interface devices.

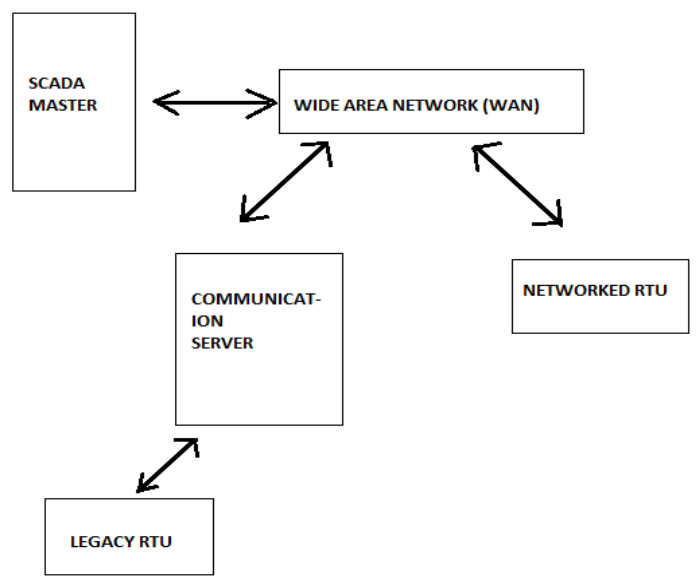

Figure 1. The architecture of Scada System

\section{The design of online monitoring system}

The Circuit Breaker monitoring system is a real time supervision system of the Circuit Breaker main parameters (currents, voltages, pressures, temperatures, contacts, etc.). This supervision is made through digital equipments and special sensors that are installed in the Circuit Breaker. The data are collected and processed in a data acquisition and control unit (UAC), to thereafter through a communication network, using desirably a protocol standardized internationally, be sent to a central computer located at the control building of the substation and later to the operation centers and so allowing a remote supervision. In the supervision computer it is installed a database manager program that stores the information history collected during the Circuit Breaker operation. A SCADA program that aside of collecting periodically the information provided by the UAC, maker possible the IHM between the monitoring system and the substation operator. A dedicated program of supervision that allows to transform the data of the historical database in information, continually making calculations and presenting the state of the Circuit Breaker monitored components, in the form of waveforms, graphs or charts, in a way to make possible an evaluation and objective analysis of the Circuit Breaker real condition, which will guide the programming of its maintenance. It is the main objective of the system, the minimization of the equipment amount of faults and the reduction of the number of programmed maintenances during the useful life.

\subsection{Implementation of Circuit Breaker monitoring system}

Digital input: They are signals that are collected continually especially in the moment of the Circuit Breaker operation. Depending on the nature of the signal, these can be collected with fast resolution (High Sampling - HS) or slow (Low Sampling - LS). HS is used when we will make the recording events, for determination of the operation times and for the comparison with obtained values of other substation monitoring equipment. The signals LS are usually used as alarms, such as information of the state of the pressure levels and levels of voltages supply DC and AC, and their resolution is in the order of seconds.

Analog input: The monitoring of the electric variables comes with two needs: one with low resolution of time (Low Sampling - LS), being necessary and enough to collect in minutes intervals, usually used for operation condition variables and the other with high resolution (High Sampling - HS) collected during the 
Circuit Breaker operation. Those events are fast so that with a high sampling rate, it's possible to make a correct oscillography of the variable and in that way to observe any type alterations inside of the normal pattern of operation.

\subsection{Monitoring system architecture}

Architecture of the system is divided into two parts: hardware and software. Hardware parts consist group of devices (transducers, converters and sensors) that are linked to an IED (Intelligent Electronics Devices) that makes the collection, treatment and subsequent sending of the data to the central computer that processes, stores and accomplishes the Interface Human Machine function (IHM). The IHM converts the data in useful information to the Circuit Breaker real condition analysis and for the communication are used standard protocols. Software part executes automated analysis of field recorded data associated with operations of group of breakers in the same switching action.

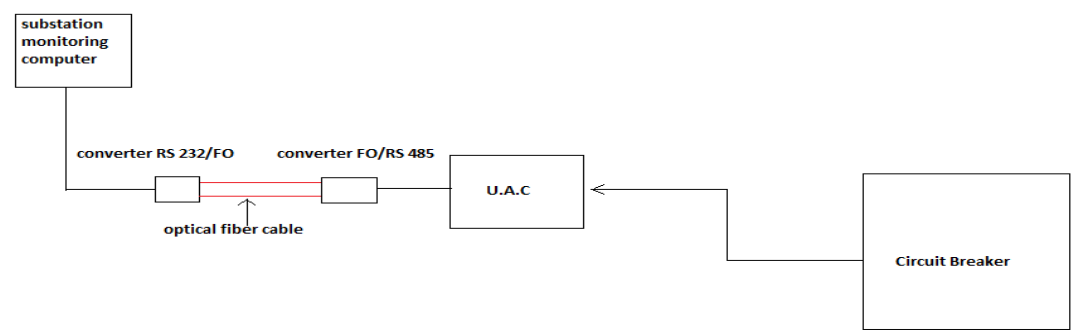

Figure 2. General architecture of circuit breaker online monitoring system

- Acquisition module

This module is composed by an IED with $240 \mathrm{kB}$ of memory configurable, a power supply of $125 \mathrm{Vdc}$ with $24 \mathrm{Vdc}$ output for feeding possible sensors, data transmission rate from 9600 to $19200 \mathrm{bps}$, Ethernet communication card, and two EIA RS-232/RS-485 ports. Acquisition Card (I/O) of binary inputs with $12 / 24 / 125 \mathrm{Vdc}$ feeding and analogical inputs of $+/-10 \mathrm{Vdc}$ or $0-20 \mathrm{~mA}$ with 16 bits resolution and accuracy of $0.1 \%$. The IED is prepared to work in atmospheres with high noise level originated by the electromagnetic fields and to support environment temperature appropriate to the substation historical temperatures.

- Digital Transducer

They are transformer units for adaptation of the line currents and voltages (phases A, B and C), with configurable inputs for one or three phase circuits. The inputs for these units are voltage from 0 to $115 \mathrm{~V}$ and current from 0 to $5 \mathrm{~A}$, being these connected through CTs and PTs of line bay. The transducers are connected to IED monitoring through the acquisition card analogical inputs ( 0 to $5 \mathrm{Vdc}$ ).

- Sensor specification

Temperature sensor: Model Pt-100 class B / RTD, this sensor is usually fixed in the own CB panel of command hold a temperature transducer for 4 to $20 \mathrm{~mA}$ output, power supply of 10 to $30 \mathrm{Vdc}$, connection to two wire, with protection tube in stainless steel, measurement of $0^{\circ} \mathrm{C}$ to $+85^{\circ} \mathrm{C}$ and accuracy of $+/-0.5 \%$. Another sensor with the same characteristics is used for measurement of the environment temperature and will be installed close to one of the poles.

Density Sensor: Model S-10, with pressure measurement from 0 to 10 bar with temperature compensation, two wires circuit, power supply from 10 to $30 \mathrm{Vdc}$, output signal from 4 to $20 \mathrm{~mA}$, pressure connection $1 / 2$ " BSP, accurately of $+/-0.5 \%$ and time response smaller than $1 \mathrm{~s}$.

Pressure Sensor: Model S-40, with pressure measurement from 0 to 400 bar, two wires circuit, power supply from 10 to $30 \mathrm{Vdc}$, output signal from 4 to $20 \mathrm{~mA}$, pressure connection $1 / 2$ "BSP, accurately of $+/-0.5 \%$ and time response smaller than $1 \mathrm{~s}$.

- Current Transducers: Type close loop (compensated) of Hall effect, with measurement variation from 0 to $10 \mathrm{~A}$ (DC or AC), three wires circuit with power supply from 20 to $30 \mathrm{Vdc}$, output signal from 4 to $20 \mathrm{~mA}$, connectionless physics used for measurement of the coil and motor currents, accuracy of $+/-0.5 \%$ and time response smaller than $1 \mathrm{~s}$. 
- Computer Monitoring Server

It consists of a microcomputer, located in the utilities substation control building, PC of $2 \mathrm{GHz}$, with $256 \mathrm{MB}$ RAM memory, 40GB HD unit, monitor 17" with card graphical interface AGP and communication cards with RS-232 ports. The SCADA system, a database management program, as well as a program developed for analysis and data presentation showing the results in form of graphs, tables, waveforms, among others.

- Communication

The needs of data communications of the Circuit Breaker monitoring system, although they don't involve a great volume of data. Different times of scanning, most of the digital and analogical signals are collected every second, while some others demand a resolution of milliseconds. The volume of data to be transmitted is usually small, except when it occurs a maneuver of the circuit breaker. The equipment collecting the data, need to be synchronized. Manufacturers offer a great variety of data communication protocols with support to those functionalities, but many of them are solutions supported just by the market products of the company, which hinders, or even, prevents its integration with other system equipment or even with SCADA.

\section{Conclusion}

The online circuit breaker monitoring system can be used to determine the condition of a specific circuit breaker, to access the condition of the circuit breaker support and to control functions, $\square$ optimize maintenance activity. This paper helps to understand how to reduce the failure rate of circuit breaker. This system is widely used in power system to control and protect power equipments under the condition of normal overload, short circuit and other faults.

\section{References}

[1] Kuncan CHEN, Wei WANG, Pinghao NI, Lijie XU,study of online monitoring system of high voltage circuit breaker, IEEE,2010

[2] Mohamed Awad and Awad I Ibrahim, PC-Based SCADA Simulator for Distribution System Analysis, IEEE

[3] Fan Xing-ming, Tang Zhuan-min, Yang Jia-zhi, He Jia-min, Huang Zhi-chao,Zhang Xin,Zou Ji-yan Determination of Arcing Duration for High-voltage Circuit Breakers Electrical Endurance On-line Monitor Based on Electromagnetic Induction, IEEE, 2010

[4] Power circuit breaker theory and design. Peter Peregrinus Ltd.

[5] SCADA-Supervisory Control and Data Acquisition, Stuart A. Boyer, Isa 2009 